\title{
Noise-Induced Excitability in Oscillatory Media
}

\author{
E. Ullner, ${ }^{1}$ A. Zaikin, ${ }^{1}$ J. García-Ojalvo, ${ }^{2,3}$ and J. Kurths ${ }^{1}$ \\ ${ }^{1}$ Institut für Physik, Potsdam Universität, Am Neuen Palais 10, D-14469 Potsdam, Germany \\ ${ }^{2}$ Center for Applied Mathematics, Cornell University, 657 Rhodes Hall, Ithaca, New York 14853, USA \\ ${ }^{3}$ Departament de Física i Enginyeria Nuclear, Universitat Politècnica de Catalunya, Colom 11, E-08222 Terrassa, Spain
}

(Received 28 May 2003; published 31 October 2003)

\begin{abstract}
A noise-induced phase transition to excitability is reported in oscillatory media with FitzHughNagumo dynamics. This transition takes place via a noise-induced stabilization of a deterministically unstable fixed point of the local dynamics, while the overall phase-space structure of the system is maintained. Spatial coupling is required to prevent oscillations through suppression of fluctuations (via clustering in the case of local coupling). Thus, the joint action of coupling and noise leads to a different type of phase transition and results in a stabilization of the system. The resulting regime is shown to display characteristic traits of excitable media, such as stochastic resonance and wave propagation. This effect thus allows the transmission of signals through an otherwise globally oscillating medium.
\end{abstract}

DOI: 10.1103/PhysRevLett.91.180601

PACS numbers: 05.40.-a, 05.70.Fh

Excitable systems are highly sensitive to perturbations, which trigger large-amplitude spiking responses above a small threshold. Noise, in particular, exerts an important influence in their dynamics. An optimal amount of noise, e.g., induces in them a coherent output in the form of roughly periodic spike trains, provided the random fluctuations are large enough to excite the system as soon as the refractory time from the previous spike is over (but not so large that the phase-space structure is destroyed) [1]. This effect, known as coherence resonance, has been observed in physical, chemical, and biological systems [2], making them behave effectively as oscillators.

Further investigations have shown that parametric noise is able to induce a bona fide transition from an excitable to an oscillatory regime, via a renormalization of the parameters defining the local dynamics of the system [3,4]. This mechanism has also been found responsible for inducing excitability in bistable [5,6] and subexcitable [3,7] media. In all those cases, however, noise has the expected role of increasing dynamical instability. In this Letter we show, on the other hand, that certain types of noise operate in the opposite direction of constructive influence, namely, enhancing stability in the system. In particular, we demonstrate that random fluctuations can induce a transition from oscillatory to excitable behavior, by stabilizing a deterministically unstable fixed point of the dynamics, while preserving the overall phase-space structure that leads to large-amplitude pulses (but which will then be triggered only by above-threshold perturbations). In contrast to previous results on noise-induced excitability, spatial coupling is absolutely essential in this case, in order to prevent noisedriven oscillations from exciting the system and converting it back in an oscillator. In that sense, the coupling plays a role similar to that of standard phase transitions, suppressing fluctuations and coupling the stable regions. It prevents the system from visiting the whole available phase space and locks it close to the stable steady state (until a perturbation triggers an excitable spike). Noiseinduced phase transitions between homogeneous phases have long been known to use the joint action of coupling and noise in this way [8,9]; here we extend this fundamental mechanism to the field of excitable dynamics.

To demonstrate this noise-induced excitability (NIE) we consider a system of coupled FitzHugh-Nagumo (FHN) elements in the oscillating state and under the action of multiplicative noise. The mechanism of a noise-induced phase transition is explained theoretically in the framework of a small-noise expansion, which extracts the systematic contribution of the multiplicative noise accounting for the excitability restoration. The excitable character of the noise-induced regime is demonstrated by showing the existence of stochastic resonance and wave propagation through the system.

Model.-We analyze the following set of $N$ coupled FHN oscillators:

$$
\begin{gathered}
\dot{u}_{i}=\frac{1}{\varepsilon}\left(F\left(u_{i}\right)-v_{i}\right)+D_{u}\left(\bar{u}_{i}-u_{i}\right), \\
\dot{\boldsymbol{v}}_{i}=c u_{i}+d+v_{i} \xi_{i}+D_{v}\left(\overline{\boldsymbol{v}}_{i}-v_{i}\right),
\end{gathered}
$$

where $\bar{x}_{i} \equiv \frac{1}{N} \sum_{j=1}^{N} x_{j}, x_{i}=u_{i}, v_{i}$, and $F(u)$ is given by

$$
F(u)= \begin{cases}-1-u+b, & u \leq-\frac{1}{2}, \\ u+b, & -\frac{1}{2}<u<\frac{1}{1+a}, \\ +1-a u+b, & u \geq \frac{1}{1+a} .\end{cases}
$$

In a neural context, $u(t)$ represents the membrane potential of the neuron and $v(t)$ is related to the time-dependent conductance of the potassium channels in the membrane [10]. The dynamics of the activator variable $u$ is much faster than that of the inhibitor $v$, as indicated by the small time-scale-ratio parameter $\varepsilon$. Coupling is considered in both the activator and the inhibitor and is taken to be global, although as we will show later, similar 
results are obtained for local diffusive coupling. Random fluctuations are represented by the $\delta$-correlated Gaussian noise $\xi_{i}(t)$, with zero mean and the correlation $\left\langle\xi_{i}(t) \xi_{j}\left(t^{\prime}\right)\right\rangle=\sigma_{m}^{2} \delta\left(t-t^{\prime}\right) \delta_{i, j}$. This multiplicative noise term is interpreted in the Stratonovich sense [9].

Phase transition to excitability.-The multiplicative noise term $v_{i} \xi_{i}$ in Eq. (2) has a nonzero mean given by $\left\langle v_{i} \xi_{i}\right\rangle=\left(\sigma_{m}^{2} / 2\right) v_{i}$ [9]. Therefore, in the presence of fluctuations the effective local dynamics of the inhibitor variable is given by $\dot{\boldsymbol{v}}_{i}=c u_{i}+d+\left(\sigma_{m}^{2} / 2\right) v_{i}$, at first order in the noise intensity. The corresponding nullclines of an isolated oscillator for increasing multiplicative intensity are represented in the phase plane of Fig. 1 (left panel). Without noise the nullcline for the slow variable $v$ (curve 1) crosses the nullcline of the fast variable (inverted-N piecewise line) in its middle segment, so that the crossing point is an unstable steady state and the system exhibits an oscillatory behavior. An increase of the multiplicative noise intensity $\sigma_{m}^{2}$ leads to a tilting and shifting of the $v$ nullcline [curves 2-4 in Fig. 1 (left panel)]. As a result, for large enough $\sigma_{m}^{2}$ (here for $\sigma_{m}^{2} \gtrsim$ 0.033 ) the crossing occurs in the left segment of the $u$ nullcline and the fixed point becomes stable. Throughout this process, however, the overall phase-space structure of the system is not changed, which allows perturbations of the noise-induced stable fixed point to excite large-amplitude excursions towards the right segment of the $u$ nullcline (excited branch). In particular, in an isolated oscillator, perturbations due to the noise itself may induce a stochastic limit cycle which prevents the system from escaping out of the oscillatory regime, i.e., the transition to excitability cannot be observed in isolated oscillators, in spite of the renormalization of the dynamical parame-
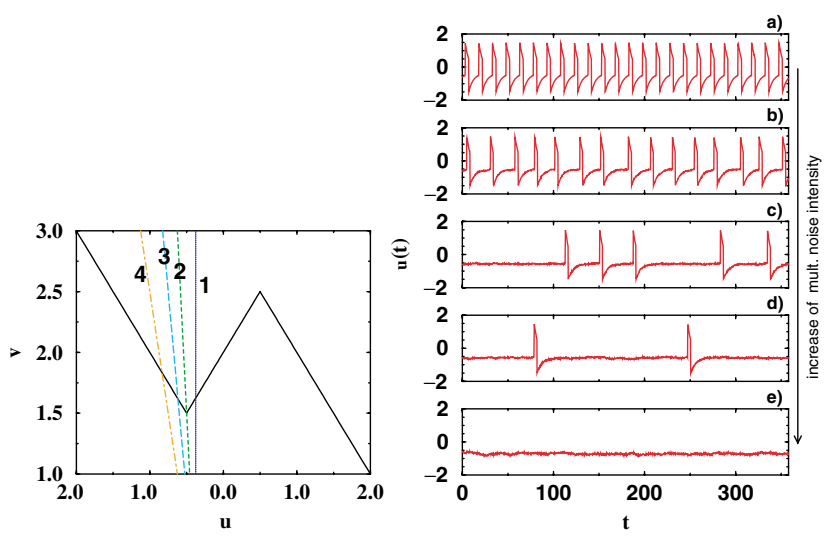

FIG. 1 (color online). Left panel: nullclines of a single FHN oscillator in phase space. The inverted-N piecewise line corresponds to the noise independent nullcline of the activator $u$. The other lines (1-4) describe the tilting of the inhibitor nullcline by increasing the noise intensity: $1-\sigma_{m}^{2}=0.0 ; 2-\sigma_{m}^{2}=$ $0.0334 ; 3-\sigma_{m}^{2}=0.06 ; 4-\sigma_{m}^{2}=0.1$. Right panel: time series of the mean field of the fast variable $u$ with increasing the multiplicative noise intensity: (a) $\sigma_{m}^{2}=0.0$; (b) $\sigma_{m}^{2}=0.033$; (c) $\sigma_{m}^{2}=0.045$; (d) $\sigma_{m}^{2}=0.05$; and (e) $\sigma_{m}^{2}=0.08$. Other parameters are $a=1.0, b=2.0, c=0.2$, and $d=0.075$. ters due to noise. In the presence of coupling, the weight of those oscillators that are not firing prevents these noiseinduced excursions and leads to effective excitability.

Figure 1 (right panel) depicts the appearance of NIE, by plotting the time series of the activator's mean field, $u(t)=(1 / N) \sum_{i=1}^{N} u_{i}(t)$ for a system of 500 coupled elements, with the coupling strengths $D_{u}=100$ and $D_{v}=$ 100 and the time-scale-ratio parameter fixed to $\varepsilon=0.01$. Because of the relatively large values of the coupling strengths (which nevertheless have the order of magnitude of $\varepsilon^{-1}$ ), the time series of the single oscillators differs only very slightly from that of the mean field, i.e., the oscillators are synchronized. Figure 1(a) displays the regular self-sustained oscillations of the system without noise. Increasing the noise intensity $\sigma_{m}^{2}$ leads to an increase and randomization of the time interval between consecutive spikes, as seen in Figs. 1(b)-1(d). Finally, for large enough noise no spike appears [Fig. 1(e)]. This corresponds to an oscillation suppression due to multiplicative noise: the system stays at the noise-induced stable fixed point. But besides an oscillation suppression, the system also exhibits excitable properties when perturbations (other than the stabilizing noise) affect the system. As shown below, this noise-induced regime displays stochastic resonance when driven periodically and wave propagation by local coupling.

In order to describe quantitatively the transition towards excitability, we compute the relative resting time with respect to the whole measuring time. Because of the random character of the time series, we need to specify a measurement threshold. We define the resting time as the interval during which every oscillator fulfills the conditions $u_{i}<-0.5$ and $v_{i} \leq 1.85$. The first requirement corresponds to the absence of spikes, and the second checks the absence of large excursions towards the left on the left branch of the $u$ nullcline. Such excursions would lead to a large excitation threshold and weaken the system's excitability. The threshold for the relative resting time is set to 0.98 .

According to the previous definitions, Fig. 2(a) displays a phase diagram in the plane of parameters $D_{u}-\sigma_{m}^{2}$ distinguishing the regions where the original oscillatory behavior and the NIE regime exist. The left boundary of the NIE balloon corresponds basically to the condition $u_{i}<-0.5$ and the right one to $v_{i}<1.85$. The NIE region shrinks in size as $D_{v}$ decreases (results not shown). In other words, minimum coupling strengths of both the activator and the inhibitor are required for the NIE regime to exist. Figure 2(b) pictures the dependence of the transition to NIE on the number of coupled elements of the system. As in standard phase transitions, the region of noise intensity values for which NIE exists becomes larger as the number of oscillators increases. We can also see that a minimum number of elements is needed (in the present case $\sim 250$ ).

Stochastic resonance in NIE.-Defined as the enhanced response to an external signal for an optimal 


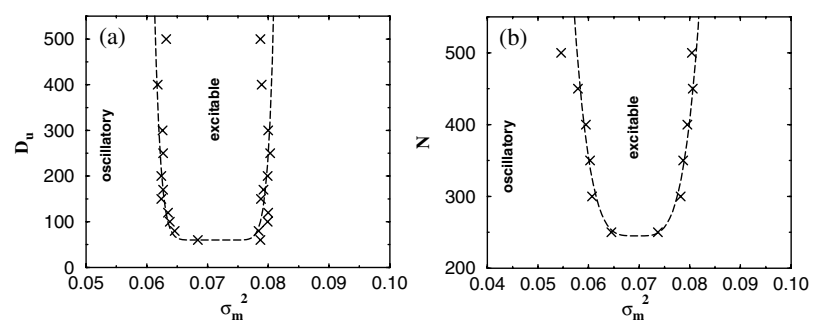

FIG. 2. Phase diagram for the transition from a self-sustained oscillatory regime to NIE. (a) Coupling strength $D_{u}$ versus multiplicative noise intensity $\sigma_{m}^{2}$ for 300 coupled elements. (b) Number of coupled elements $N$ versus multiplicative noise intensity $\sigma_{m}^{2}$ for $D_{u}=416$ and $D_{v}=64$.

amount of noise, stochastic resonance (SR) has long been found in excitable media [11-13]. In order to show that the NIE regime possesses all immanent properties of excitable systems, we now examine the response of the system to external periodic driving and an additive source of noise. The behavior of the inhibitor is then given by

$$
\dot{\boldsymbol{v}}_{i}=c u_{i}+d+v_{i} \xi_{i}+D_{v}\left(\bar{v}_{i}-v_{i}\right)+\zeta_{i}+f \cos \omega_{f} t,
$$

where $\zeta_{i}$ is a Gaussian white noise with intensity $\sigma_{a}^{2}$, the intensity of the multiplicative noise is taken large enough to make the system excitable, and the amplitude $f$ of the external forcing is chosen small enough so that no excitation is produced in the absence of the additive noise. We are interested in the response of the system at the signal frequency $\omega_{f}$ when the additive noise intensity $\sigma_{a}^{2}$ is increased. Figure 3 (left panel) displays the time series of the averaged activator concentration for different additive noise intensities, superimposed with the periodic input signal (with enlarged amplitude for a better comparability with the output signal). In the absence of additive noise [Fig. 3(a)], the signal alone is too small, and the system remains at the noise-induced stable fixed point. When additive noise is added, spikes appear more and more frequently, until at an optimal noise intensity the spikes occur basically synchronously with the signal [Figs. 3(b)-3(d)]. Further increase of additive noise destroys the synchronization effect [Fig. 3(e)].

To evaluate the linear response $Q$ of the system at the input frequency $\omega_{f}$ we extract the parameter $Q$ from a signal $\left\langle u_{r}\right\rangle$ as in $[14,15]$. In order to compute this quantity, we neglect subthreshold dynamics and replace the global signal by $\left\langle u_{r}(t)\right\rangle=\Theta\left(\langle u\rangle-u_{\mathrm{th}}\right)-0.6 \Theta\left(u_{\mathrm{th}}-\langle u\rangle\right)$, where $\langle\cdots\rangle$ denotes the average over the population and $u_{\mathrm{th}}=-0.45$. The numerical results are shown in Fig. 3 (right panel) both with and without the multiplicative noise. The typical bell-shaped SR curve appears only in the presence of a suitable multiplicative noise intensity (i.e., in the NIE regime), whereas in the original selfsustained oscillatory regime $\left(\sigma_{m}^{2}=0.0\right)$, the SR effect cannot be observed. The former behavior corresponds to a double stochastic effect [15-18], because optimal response in the presence of additive noise occurs due to a
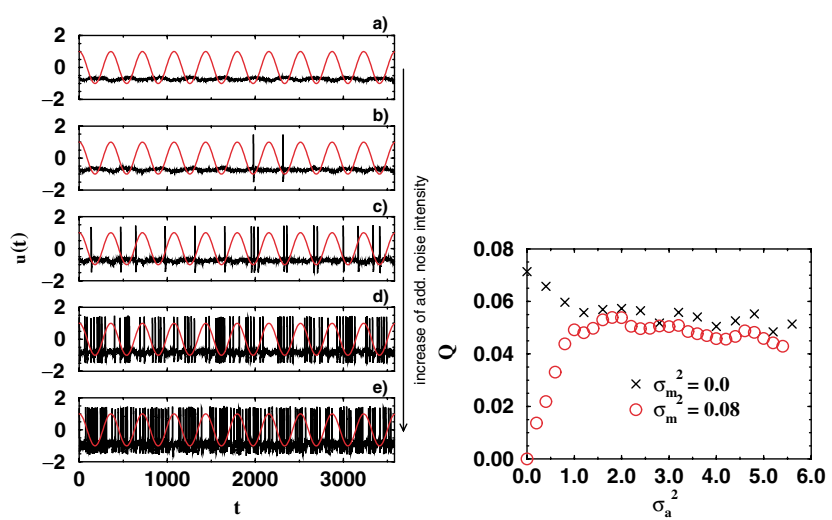

FIG. 3 (color online). Left panel: time series of the average activator concentration for increasing additive noise intensity and $\sigma_{m}^{2}=0.08$ : (a) $\sigma_{a}^{2}=0.0$; (b) $\sigma_{a}^{2}=0.1$; (c) $\sigma_{a}^{2}=0.5$; (d) $\sigma_{a}^{2}=2.0$; and (e) $\sigma_{a}^{2}=5.0$. Right panel: response of the system to the signal frequency versus additive noise intensity, for $\sigma_{m}^{2}=0.08$ (circles) and $\sigma_{m}^{2}=0.0$ (crosses). Parameters are $D_{u}=100, D_{v}=100, f=0.012$, and $\omega_{f}=0.0175$. Other parameters are those of Fig. 1.

property (excitability) which is induced by a second, multiplicative noise. In light of these results, one could speculate that sensory adaptation by noise in living organisms [19] can be possible even in oscillatory situations because parametric noise can suppress undesirable oscillations.

Wave propagation in NIE.-One of the main characteristics of excitable media is their ability to sustain propagation of structures. This is, e.g., the way in which electrical pulses propagate through neural tissue in physiological systems [10]. The NIE regime reported here offers the possibility of a signal propagation through oscillatory media. Additionally, NIE allows the activation/deactivation of the excitable property so that information transport can be controlled by multiplicative noise.

In order to verify that the NIE regime allows the propagation of excitable structures, we substitute the global coupling considered so far by a local diffusive coupling. Hence the coupling term in Eqs. (1) and (2) is now given by $\bar{x}_{i} \equiv \frac{1}{\mathcal{N}} \sum_{j \in n . n} x_{j}$, where the sum runs only over the $\mathcal{N}$ nearest neighbors of site $i$. In what follows we consider a 2-dimensional lattice with fixed boundary conditions. Additionally, the $u$ nullcline is now given by

$$
F(u)= \begin{cases}-1-u+b, & u \leq-\frac{1}{2}, \\ g u+b+\frac{1}{2}(g-1), & -\frac{1}{2}<u<\frac{1}{g}-\frac{1}{2}, \\ +1-a u+b-\frac{1}{2}+a\left(\frac{1}{g}-\frac{1}{2}\right), & u \geq \frac{1}{g}-\frac{1}{2},\end{cases}
$$

in such a way that the slope of its unstable middle branch decreases, so that the refractory time becomes smaller. The dynamical equation of $v$, on the other hand, is unchanged from Eq. (2), and thus the noise-induced transition mechanism described above persists. Under these conditions, this system displays a noise-induced phase 

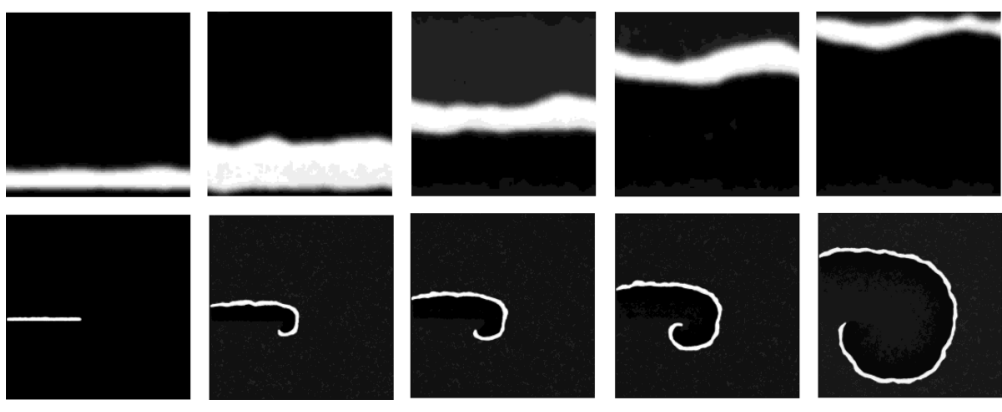

FIG. 4. Snapshots of the activatory variable $u$ for increasing time (from left to right). The upper row shows the propagation of a plane wave front in an $130 \times 130$ array at time steps 10.9, $11.5,12.3,13.4$, and 14.2 time units. The lower row shows spiral wave propagation in a $1000 \times$ 1000 array at time steps $11.0,13.5,14.5,16.0$, and 21.0 time units. Parameters are $\varepsilon=0.01$, $a=1.0, b=2.0, c=0.2, d=0.075, g=0.2, D_{u}=416, D_{v}=64$, and $\sigma_{m}^{2}=0.072$.

transition to excitability, as for global coupling, but in this case via the formation of clusters of stable elements. In the NIE region (i.e., for large enough $\sigma_{m}^{2}$ ), independently of the initial conditions every oscillator of the coupled ensemble moves to the NIE fixed point and remains there. In this situation, the spatiotemporal response of the system to a plane-wave perturbation is depicted in Fig. 4 (upper row). We observe a clear propagation of the plane wave from bottom to top. Spiral wave propagation can also be seen in Fig. 4 (lower row). In the absence of multiplicative noise, on the other hand, the system exhibits a synchronous self-sustained oscillatory behavior and no wave propagation can be observed. This means that the presence of multiplicative noise is crucial for information transmission in this system.

In summary, we have studied a different kind of phase transition in which the application of noise to an array of oscillating elements leads to the suppression of oscillations and induces excitability. The appearance of noiseinduced excitability is a collective effect and occurs via a phase transition due to the joint action of coupling and multiplicative noise. In contrast to standard phase transitions and other studies on excitable systems [3-7], the increase of noise enhances the stability in the system and restores excitable properties. This noise-supported excitability displays characteristic properties of standard excitable media, such as stochastic resonance and wave propagation. Since SR relies on a property of the system which is in turn induced by noise, optimization of both noise sources is needed, and hence this effect is an example of a doubly stochastic phenomenon [16]. The interplay between excitable and oscillatory dynamics in noisy systems is a current important issue [20]. In particular, these theoretical findings suggest a possible mechanism to suppress undesirable global oscillations in neural networks (which are usually characteristic of abnormal medical conditions such as Parkinson's disease or epilepsy), using the action of noise to restore excitability, which is the normal state of neuronal ensembles.

E. U. acknowledges the International MP Research School on Biomimetic systems, E. U. and A. Z. CESCA-
CEPBA (the EC IHP Program No. HPRI-1999-CT00071), J.G. O. DGES (Spain, BFM2001-2159 and BFM2002-04369), and J. K. SFB 555 (Germany).

[1] A. Pikovksy and J. Kurths, Phys. Rev. Lett. 78, 775 (1997).

[2] B. Lindner et al., Phys. Rep. (to be published).

[3] S. Alonso et al., Phys. Rev. Lett. 87, 078302 (2001).

[4] J. García-Ojalvo, F. Sagués, J. M. Sancho, and L. Schimansky-Geier, Phys. Rev. E 65, 011105 (2002).

[5] J. García-Ojalvo and L. Schimansky-Geier, J. Stat. Phys. 101, 473 (2000).

[6] R. Báscones, J. García-Ojalvo, and J. M. Sancho, Phys. Rev. E 65, 061108 (2002).

[7] S. Kádár, J. Wang, and K. Showalter, Nature (London) 391, 770 (1998).

[8] C. Van den Broeck, J. M. R. Parrondo, and R. Toral, Phys. Rev. Lett. 73, 3395 (1994).

[9] J. García-Ojalvo and J. M. Sancho, Noise in Spatially Extended Systems (Springer, New York, 1999).

[10] J. Keener and J. Snyder, Mathematical Physiology (Springer, New York, 1998).

[11] F. Moss et al., Ann. N.Y. Acad. Sci. 706, 26 (1993).

[12] K. Wiesenfeld et al., Phys. Rev. Lett. 72, 2125 (1994).

[13] B. Lindner, Coherence and Stochastic Resonance in Nonlinear Dynamical Systems (Logos-Verlag, Berlin, 2002).

[14] L. Gammaitoni, P. Hänggi, P. Jung, and F. Marchesoni, Rev. Mod. Phys. 70, 223 (1998).

[15] A. Zaikin, J. García-Ojalvo, L. Schimansky-Geier, and J. Kurths, Phys. Rev. Lett. 88, 010601 (2002).

[16] A. Zaikin, Fluctuations and Noise Letters 2, L157 (2002).

[17] A. Zaikin, J. García-Ojalvo, R. Báscones, E. Ullner, and J. Kurths, Phys. Rev. Lett. 90, 030601 (2003).

[18] A. Zaikin, J. Kurths, and L. Schimansky-Geier, Phys. Rev. Lett. 85, 227 (2000).

[19] D. Russell, L. Wilkens, and F. Moss, Nature (London) 402, 291 (1999).

[20] J. M. G. Vilar et al., Proc. Natl. Acad. Sci. U.S.A. 99, 5988 (2002). 